\title{
Predictors of Venous Thromboembolism in Patients with Advanced Common Solid Cancers
}

\author{
Isaac E. Hall, ${ }^{1}$ Martin S. Andersen, ${ }^{1}$ Harlan M. Krumholz, ${ }^{2,3,4}$ and Cary P. Gross ${ }^{1,2}$ \\ ${ }^{1}$ Sections of General Internal Medicine, Primary Care Center, Yale University School of Medicine, 333 Cedar Street, \\ P.O. Box 208025, New Haven, CT 06520, USA \\ ${ }^{2}$ Section of Cardiovascular Medicine, Department of Medicine, Robert Wood Johnson Clinical Scholars Program, \\ New Haven, CT 06520, USA \\ ${ }^{3}$ Department of Epidemiology and Public Health, Yale University School of Medicine, New Haven, CT 06520, USA \\ ${ }^{4}$ The Center for Outcomes Research and Evaluation, Yale New Haven Hospital, New Haven, CT 06520, USA \\ Correspondence should be addressed to Cary P. Gross, cary.gross@yale.edu
}

Received 23 June 2009; Revised 23 October 2009; Accepted 9 December 2009

Recommended by Hermann Brenner

\begin{abstract}
There is uncertainty about risk heterogeneity for venous thromboembolism (VTE) in older patients with advanced cancer and whether patients can be stratified according to VTE risk. We performed a retrospective cohort study of the linked MedicareSurveillance, Epidemiology, and End Results cancer registry in older patients with advanced cancer of lung, breast, colon, prostate, or pancreas diagnosed between 1995-1999. We used survival analysis with demographics, comorbidities, and tumor characteristics/treatment as independent variables. Outcome was VTE diagnosed at least one month after cancer diagnosis. VTE rate was highest in the first year (3.4\%). Compared to prostate cancer (1.4 VTEs/100 person-years), there was marked variability in VTE risk (hazard ratio (HR) for male-colon cancer 3.73 (95\% CI 2.1-6.62), female-colon cancer HR 6.6 (3.83-11.38), up to female-pancreas cancer HR 21.57 (12.21-38.09). Stage IV cancer and chemotherapy resulted in higher risk (HRs 1.75 (1.44-2.12) and 1.31 (1.0-1.57), resp.). Stratifying the cohort by cancer type and stage using recursive partitioning analysis yielded five groups of VTE rates (nonlocalized prostate cancer 1.4 VTEs/100 person-years, to nonlocalized pancreatic cancer 17.4 VTEs/100 patientyears). In a high-risk population with advanced cancer, substantial variability in VTE risk exists, with notable differences according to cancer type and stage.
\end{abstract}

Copyright (C) 2009 Isaac E. Hall et al. This is an open access article distributed under the Creative Commons Attribution License, which permits unrestricted use, distribution, and reproduction in any medium, provided the original work is properly cited.

\section{Introduction}

The morbidity and mortality associated with venous thromboembolism (VTE) are substantial $[1,2]$. Chronic and debilitating sequelae include postthrombotic syndrome following deep vein thrombosis (DVT) or chronic pulmonary hypertension due to pulmonary embolism (PE) $[3,4]$. The economic burden of VTE treatment is also quite high, causing direct hospital costs of over $\$ 3000$ per discharge in a recent analysis [5]. These considerations are further compounded for patients who develop VTE in the setting of malignancy, as cancer further increases the risk of adverse outcomes in patients with VTE [6-8].

While the association between thrombosis and cancer was first established over 100 years ago, the independent impact of VTE on patients with cancer has been more recently described [9]. VTE is associated with up to $40 \%$ increased risk of death in patients with cancer, while in certain cancer types, the mortality associated with VTE is negligible [7]. The same analysis revealed an excess risk of hemorrhage associated with VTE in this population as high as $11.5 \%$, depending on cancer type [7]. The adverse effect of VTE on patients and the morbidity of prevention and treatment strategies reinforce the need to identify which cancer patients are at highest risk of VTE. High-risk groups can be monitored more closely for VTE and might be more likely to benefit from primary thromboprophylaxis.

Prior work has described substantial variability in VTE incidence in cancer patients based on cancer type and other characteristics. Certain groups of patients with cancer are at no greater risk for VTE than the general population, while other groups have very high risk $[8,10-12]$. Additional work 


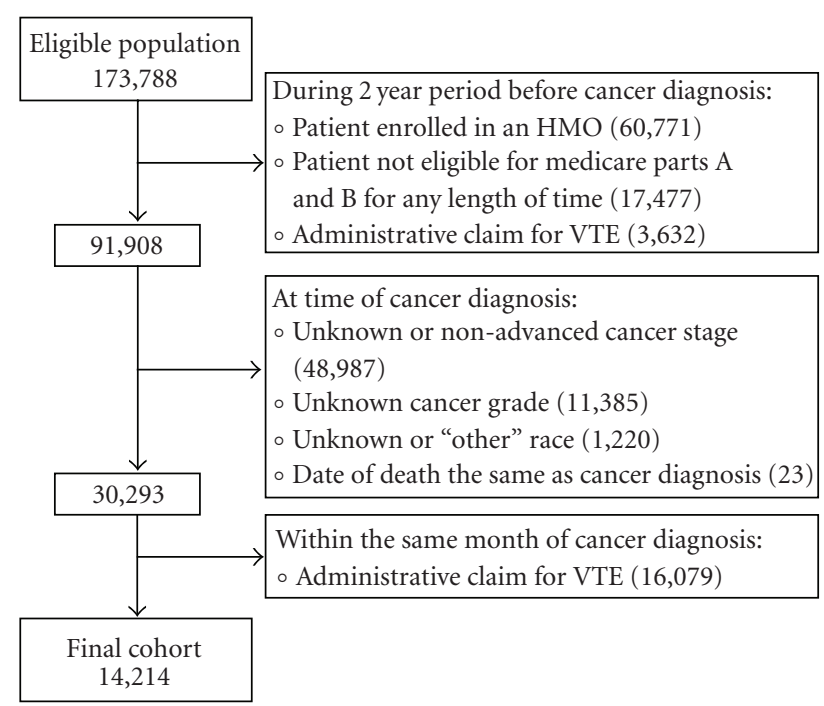

FIGURE 1: Construction of the cohort. The eligible population included patients listed in the SEER-Medicare Database who were diagnosed (between 1995 and 1999) with incident stage III or IV breast, lung, or colon cancer, or with nonlocalized cancer of the prostate or pancreas at the age of 67 years or older.

has demonstrated that stage and time since diagnosis were strongly associated with VTE risk, although important confounders such as cancer therapy and comorbid conditions have not been fully investigated [6]. There also remains a significant knowledge gap regarding the variability of VTE risk within the most advanced, metastatic stages of cancer. Furthermore, it is not understood whether traditional risk factors within the general population continue to be effect modifiers of VTE risk in patients with very advanced cancer. The goal of this study was to better delineate important risk factors within a vulnerable population traditionally believed to be at very high risk for VTE and to assess the degree of variability in VTE incidence across risk strata.

Trials of primary prophylaxis in the cancer patient population have been plagued by low event rates, suggesting that despite the diagnosis of malignancy, many of these patients had low risk for VTE $[13,14]$. There are also very little data regarding the best way to stratify patients with malignancy into clinically relevant subgroups based on VTE risk. Better knowledge of risk could inform decision-making regarding future study design and the decision to employ thromboprophylaxis in this high-risk patient population. We therefore performed a retrospective, population-based cohort study of patients with five common solid tumors that were diagnosed at advanced stages, using a nationally representative database, to investigate the variability and incidence of VTE and to demonstrate an effective risk stratification approach.

\section{Methods}

2.1. Data Source and Study Sample. We identified the cohort using the Surveillance, Epidemiology, and End Results (SEER) Medicare Database, which provides detailed patientand tumor-level information, including socioeconomic sta- tus of the patient, as well as stage, grade, and location of the tumor. This population-based database collects information from specific geographic locations and represents $26 \%$ of the entire United States population. For those over the age of 65, Medicare claims are also available and have been linked to the SEER database at the patient level [15-17].

The study sample consisted of patients diagnosed with incident stage III or IV breast, lung, or colon cancer, or with nonlocalized cancer of the prostate or pancreasselected because more patients die of these cancers each year than any others $[18,19]$. The study population was restricted to patients diagnosed with cancer from 1995 through 1999, given that outpatient treatment of VTE was relatively uncommon during this time. In order to have two years of prediagnosis claims, we only considered patients 67 years old or older when diagnosed with cancer, yielding an eligible population size of 173,788 patients (Figure 1). We excluded those who at anytime during the two years prior to their cancer diagnosis were not eligible for Medicare Parts A and B $(17,477)$ or had enrolled in an HMO $(60,771)$. Patients diagnosed with cancer at the time of death (23), those with an unknown cancer grade $(11,385)$, an unknown or nonadvanced cancer stage $(48,987)$, and those listed as unknown or "other" race $(1,220)$, were also excluded. Finally, in order to capture patients who did not have a VTE diagnosed prior to or concomitantly with their cancer diagnosis, we excluded patients who had an administrative claim consistent with VTE during the two years prior to $(3,632)$ or within the same month of their cancer diagnosis $(16,079)$.

2.2. Construction of Variables. We defined VTE as either DVT or PE using International Classification of Diseases, 9th Revision, Clinical Modification (ICD-9-CM) claims codes: 451.1, 451.11, 451.19, 451.81, 453.2, 453.3, 453.8, 453.9, 415.1, 415.11, and 415.19. We included only those VTEs 
TABLE 1: Sample characteristics by cancer type.

\begin{tabular}{lccccccccc}
\hline Cancer Type & No. & Sex, \% F & Median Age, yr & Race, \% White & \% High Grade & \% Stage IV & \% Surgery & \% Chemo & \% Radiation \\
\hline Breast & 1,789 & 100 & 76 & 89.6 & 58 & 37.1 & 80.5 & 34.4 & 37.1 \\
Colon & 1,890 & 57.2 & 75 & 93 & 27 & 37.4 & 87.3 & 42.2 & 36.4 \\
Lung & 8,102 & 43.4 & 74 & 91.7 & 78.5 & 50.5 & 18.2 & 38.6 & 53.7 \\
Prostate & 1,596 & 0 & 76 & 85 & 62.1 & 100 & 24.9 & 46.4 & 17.2 \\
Pancreas & 837 & 56 & 75 & 89.7 & 53.8 & 100 & 12.5 & 33.8 & 10.9 \\
\hline
\end{tabular}

which were associated with a specific hospital admission. The date of VTE was defined as the date of a hospitalization with a primary or secondary ICD-9-CM diagnosis code for DVT or PE. VTE more than likely necessitated admission in the vast majority of cases during our study period, given that the first FDA approval of a drug for outpatient treatment of VTE did not occur until the final year of the study period. Thus, any VTE diagnosis code recorded in the outpatient setting likely referred to an old VTE (prior to cancer diagnosis), a prior VTE recorded in a system not linked with Medicare (i.e., the Veteran's Administration), or was possibly a miscode. Hence, in order to enhance specificity, only patients with inpatient VTE ICD-9 codes were assigned a VTE diagnosis. For each patient, we created indicators for histological grade (with grades I and II defined as "low grade" and grades III and IV defined as "high grade"), cancer specific surgery, chemotherapy, radiation therapy, central venous catheters, and one indicator for each condition comprising the Charlson comorbidity index, with the exception of cancer [20]. We also included appropriate ICD-9 codes for hip fracture and atrial fibrillation as we hypothesized that these conditions or their treatments (such as anticoagulation) might affect the risk of VTE [21-23]. These variables were obtained from hospital, outpatient, and physician claims using previously described methods $[20,24,25]$.

2.3. Statistical Analysis. Demographic characteristics as well as cancer grade, cancer-specific surgery, chemotherapy, and radiation therapy were summarized for each cancer type. We estimated incidence rates for VTE (in events per 100 personyears) during each of the first three years after the diagnosis of cancer. We censored individuals at death, diagnosis of VTE, loss of part A or B coverage, or enrollment in an HMO. We restricted our analyses of potential predictors of VTE to the first year after cancer diagnosis, as VTE incidence was the highest during this time period. We performed bivariate analyses of gender, age, race, cancer type, stage, grade, surgery, radiation therapy, chemotherapy, central venous catheter use, and individual comorbidities versus development of VTE within one year of cancer diagnosis. We also performed a number of initial stratified analyses to better understand the basic trends in the database. Those variables with $P$ values less than or equal to .2 by nonstratified, bivariate analysis were entered into a preliminary multivariate Cox proportional hazards model, using the same censoring strategy outlined above. Variables with $P$ values less than or equal to .05 were retained in the final model. The interaction between sex and cancer type was assessed by a Wald test in the full model. Each cancer type was separated by sex in the multivariate model when appropriate (i.e., male colon, female colon, etc.) to allow all combinations of sex and cancer type to be included in the full model.

We used recursive partitioning analysis (RPA) to identify clinically distinct groups. RPA involves building binary decision trees that separate a population, based on prognostic factors, into groups with similar outcomes (development of VTE in this case). The strength of recursive partitioning is its ability to identify those factors that separate groups into the most dissimilar outcomes in a nonlinear fashion. RPA has been used with increasing frequency to identify important interactions between factors in different subgroups [26]. The subgroups that result from RPA are often more homogeneous compared with traditional multivariate regression models.

In our preliminary analysis, all variables that were significantly associated with development of VTE in the multivariate Cox model were entered into the RPA. These initial splitting algorithms consistently employed cancer type and stage of malignancy, as the first splitting "levels." In order to derive a limited number of patient strata that are clinically distinct and meaningful, the final RPA splits the cohort using only these two factors. The rate of VTE within one year of cancer diagnosis was then calculated for each group. We then calculated $P$ values for the differences between groups using Student's $t$-tests.

SAS 9.1.3 (SAS Institute Inc, Cary, NC) was used to calculate incidence rates and for survival analysis. Recursive partitioning was performed using the STREE software developed by Heping Zhang and Singer [27].

\section{Results}

Of the 14,214 patients included, the majority $(8102,57 \%)$ had lung cancer, followed by colon (1890), breast (1789), prostate (1596), and pancreas (837) (Table 1). The median age was similar across all five cancer types at about 75 years old. For patients with prostate cancer, $15 \%$ were Black, compared to $7 \%-10 \%$ of those with other cancers. The use of cancer-specific therapy varied across cancer type. The vast majority of patients with breast or colon cancer $(80.5 \%$ and $87.3 \%$, resp.) underwent surgery, while less than a quarter of the patients with the remaining malignancies had cancerspecific surgery. 


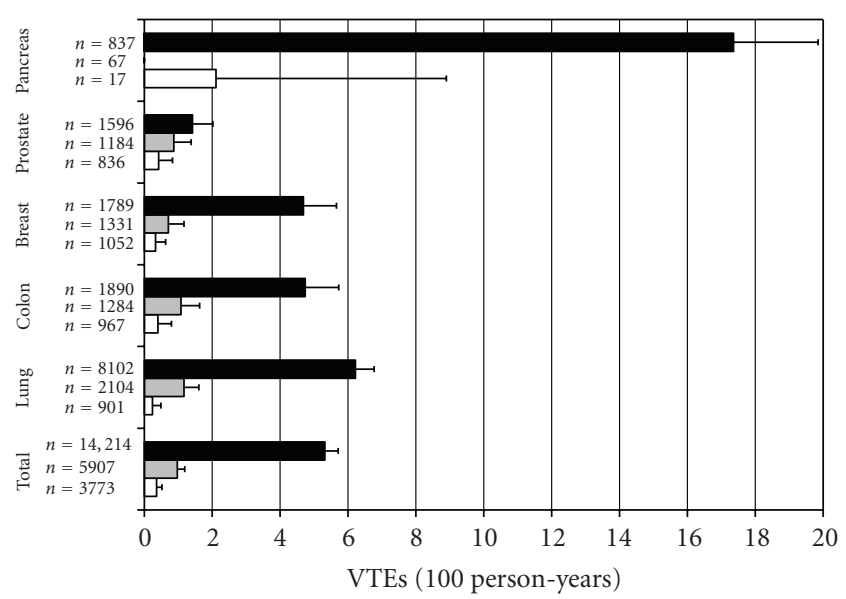

- Year 1

ㄱear 2

$\square$ Year 3

Figure 2: Incidence of VTE according to cancer type and time. Incidence of VTE within each year after cancer diagnosis is shown with error bars depicting standard deviations.

Incidence rates for VTE varied significantly by cancer type (Figure 2). Within one year of cancer diagnosis, prostate cancer had the lowest rate of VTE at 1.42 per 100 personyears, while pancreatic cancer had the highest at 17.36 per 100 person-years $(P<.01$; Figure 2$)$. The other cancers had rates ranging from approximately 4 to 6 per 100 personyears. There was a sharp decline in incidence for all cancer types in years two and three after diagnosis of cancer. The overall rate (per 100-person years) decreased from 5.32 (95\% CI 4.93-5.67) in the first year to $0.97(0.75-1.25)$ and $0.36(0.20-0.60)$ for the second and third years, respectively $(P<.0001$ for first year rate compared with both second and third year rates).

In bivariate analysis, VTE was significantly related to sex, age, cancer type, surgery, chemotherapy, and radiation therapy (Table 2). Four of the comorbid conditions were modestly associated with VTE, though in a protective fashion. These included congestive heart failure (CHF: 2.5\% of those with a CHF ICD-9 code had a VTE in the first year after cancer diagnosis versus $3.5 \%$ of those without CHF, $P=.07$ ), chronic obstructive pulmonary disease (COPD: $2.7 \%$ with COPD versus $3.6 \%$ without, $P=.02$ ), dementia $(<1 \%$ with dementia versus $3.5 \%$ without, $P=.12$ ), and atrial fibrillation $(2.2 \%$ with atrial fibrillation versus $3.5 \%$ without, $P=.04)$.

With prostate cancer as the reference in the final model, all of the other groups had increased hazard ratios (HRs) for risk of VTE in the first year after cancer diagnosis, ranging from 3.73 for male-colon (95\% confidence interval: 2.16.62) to 21.57 (12.21-38.09) for female-pancreas (Table 3). Stage IV cancer had an increased risk of 1.75 (1.44-2.12) when compared with stage III. The use of chemotherapy during the first year after diagnosis resulted in an HR of 1.31 (1.10-1.57). Increasing age trended toward protective against VTE, with those older than 85 years showing an HR of 0.66
(0.42-1.06), though none of the age groups reached a level of statistical significance.

Only one of the three cancer type-sex interactions was significant (with prostate cancer as the reference group); the risk for VTE was higher for women compared with men with colon cancer (female HR 6.6 (3.83-11.38), male HR 3.73 (2.1-6.62), $P=.02$ for sex $*$ colon cancer interaction). The VTE risk was similar for both sexes among patients with pancreatic or lung cancer.

RPA analysis resulted in five distinct VTE risk groups (Figure 3). Initial partitioning separated nonlocalized prostate cancer from the cohort with a VTE rate of 1.4 per 100 person-years. Stage III cancers were separated from the remaining cohort in the second split. This group was further divided by lung cancer with a VTE rate of 5.2 per 100 person-years, leaving breast and colon cancer, with a combined VTE rate of 4.0 per 100 person-years. The last splitting procedure separated nonlocalized pancreatic cancer, with the highest rate of 17.4 , and leaving stage IV cancer of the lung, breast, and colon with a combined rate of 7.2 VTEs per 100 person-years.

\section{Discussion}

Patients with advanced cancer are at increased risk for VTE $[6,7,28]$. We have added three specific findings to this body of knowledge using a population-based cohort of older patients with five common, advanced solid tumors. (1) Even in advanced cancer, there is significant heterogeneity in the incidence and risk for VTE within the first years after cancer diagnosis, based on cancer type and stage. (2) Increasing stage and the use of chemotherapy elevate VTE risk in advanced cancer, while other traditional risk factors are not significant in this population. (3) Effective risk stratification is possible within a population with advanced malignancy using recursive partitioning. This allows for the identification of those patients with highest VTE risk, indicating who should be most closely monitored.

Several mechanisms lead to an increased risk of VTE in the cancer patient population. The significant variability in VTE incidence and risk demonstrated here is likely related to nonuniform distribution of these mechanisms between cancer types, stages, and the use of chemotherapies. Generalized causes include more frequent use of central venous catheters, decreased mobility, and more frequent acute medical illnesses in the oncology population than in the general population. Other malignancy-associated mechanisms include direct vascular invasion and external compression by tumor, endothelial cell injury from chemotherapy, cancer cell production of tissue factor and factor X activator, platelet accumulation and activation, and increased levels of acute-phase reactants like von Willebrand factor and factor VIII due to cancer-related inflammation [29-31].

The variability in VTE risk shown in the current study bolsters previous work on VTE predictors in patients with cancer. Chew et al. demonstrated a high incidence of thromboembolism in metastatic cancer of the pancreas, stomach, 
TABLe 2: Patient and cancer characteristics and rate of venous thromboembolism within 1 year after cancer diagnosis.

\begin{tabular}{|c|c|c|c|c|c|}
\hline Predictor & Total & VTE & $\%$ & $(95 \% \mathrm{CI})$ & $P$ value \\
\hline Male & 7,579 & 219 & 2.9 & $(2.51-3.27)$ & \\
\hline Female & 6,635 & 270 & 4.1 & $(3.59-4.55)$ & $<.01$ \\
\hline Age 67-69 & 2,427 & 107 & 4.4 & $(3.59-5.23)$ & \\
\hline Age 70-74 & 4,497 & 184 & 4.1 & $(3.51-4.67)$ & \\
\hline Age 75-79 & 3,791 & 119 & 3.1 & $(2.58-3.7)$ & \\
\hline Age $80-84$ & 2,282 & 56 & 2.5 & $(1.82-3.08)$ & \\
\hline Age 85+ & 1,217 & 23 & 1.9 & $(1.12-2.66)$ & $<.01$ \\
\hline White & 12,899 & 439 & 3.4 & $(3.09-3.71)$ & \\
\hline Black & 1,315 & 50 & 3.8 & $(2.77-4.83)$ & .45 \\
\hline Breast cancer & 1,789 & 72 & 4.0 & $(3.11-4.93)$ & \\
\hline Colon cancer & 1,890 & 74 & 3.9 & $(3.05-4.79)$ & \\
\hline Lung cancer & 8,102 & 273 & 3.4 & $(2.98-3.76)$ & \\
\hline Prostate cancer & 1,596 & 20 & 1.3 & $(0.7-1.8)$ & \\
\hline Pancreatic cancer & 837 & 50 & 6.0 & $(4.36-7.58)$ & $<.01$ \\
\hline Stage 3 & 6,322 & 221 & 3.5 & $(3.05-3.95)$ & \\
\hline Stage 4 & 7,892 & 268 & 3.4 & $(3-3.8)$ & .75 \\
\hline Grade 1 or 2 & 4,867 & 171 & 3.5 & $(2.99-4.03)$ & \\
\hline Grade 3 or 4 & 9,347 & 318 & 3.4 & $(3.03-3.77)$ & .73 \\
\hline Surgery & 5,065 & 204 & 4.0 & $(3.49-4.57)$ & $<.01$ \\
\hline Chemotherapy & 5,559 & 260 & 4.7 & $(4.12-5.24)$ & $<.01$ \\
\hline Radiation therapy & 6,069 & 251 & 4.1 & $(3.64-4.64)$ & $<.01$ \\
\hline MI & 384 & 11 & 2.9 & $(1.19-4.53)$ & .53 \\
\hline $\mathrm{CHF}$ & 1,059 & 26 & 2.5 & $(1.53-3.39)$ & .07 \\
\hline PVD & 517 & 15 & 2.9 & $(1.45-4.35)$ & .49 \\
\hline CVD & 687 & 19 & 2.8 & $(1.53-4)$ & .32 \\
\hline COPD & 2,652 & 71 & 2.7 & $(2.07-3.29)$ & .02 \\
\hline Dementia & 117 & $<5$ & 0.9 & $(0-2.51)$ & .12 \\
\hline Diabetes & 1,689 & 50 & 3.0 & $(2.15-3.77)$ & .25 \\
\hline Chronic Kidney Disease & 160 & $<5$ & 1.9 & $(0-3.98)$ & .28 \\
\hline Ulcers & 213 & 6 & 2.8 & $(0.6-5.04)$ & .62 \\
\hline Rheumatologic disease & 264 & 9 & 3.4 & $(1.22-5.6)$ & .98 \\
\hline Hip fracture & 395 & 12 & 3.0 & $(1.35-4.73)$ & .66 \\
\hline Atrial Fibrillation & 896 & 20 & 2.2 & $(1.26-3.2)$ & .04 \\
\hline Obesity & 103 & 5 & 4.9 & $(0.7-9)$ & .43 \\
\hline
\end{tabular}

Bivariate analysis of cancer and patient characteristics on development of VTE. The following comorbid conditions were removed because of prevalences <100: paralysis, cirrhodites, AIDS, and central venous catheter. Myocardial infarction: MI; congestive heart failure: CHF; peripheral vascular disease: PVD; cerebrovascular disease: CVD; chronic obstructive pulmonary disease: COPD.

bladder, uterus, kidney, and lung with the highest incidence of VTE occurring in the first year after cancer diagnosis [6]. Sallah et al. noted that chemotherapy, advanced malignancy, and tumors of the kidney, pancreas, stomach, and brain were independently associated with VTE [32]. Blom et al. showed a high incidence of VTE in cancers of the bone, ovary, brain, and pancreas as well as higher VTE risk with distant metastases, chemotherapy, and hormone therapy [11]. As with our findings, the latter study did not reveal strong associations for VTE with radiotherapy or surgery. Hormonal therapy was not differentiated from chemotherapy in our database. The lower risk associated with years two and three was likely due to the fact that the most aggressive cancers lead to increased mortality in addition to increased risk of VTE, as evident by the large decrement in total patients with each successive year (Figure 2).

The absolute risk of VTE seen in this cohort is quite high in comparison to the general population. Using two population-based cohorts of patients at least 45 years old, in which less than $8 \%$ had cancer, the LITE study demonstrated a higher rate of VTE in men than women over the age of 74-5.5 versus 2.7 per 1000 person-years [33]. These rates are much lower than our reference rate of 1.4 VTEs per 100 person-years (or 14 per 1000 person-years) in patients with nonlocalized prostate cancer, with all other groups demonstrating even higher rates. 


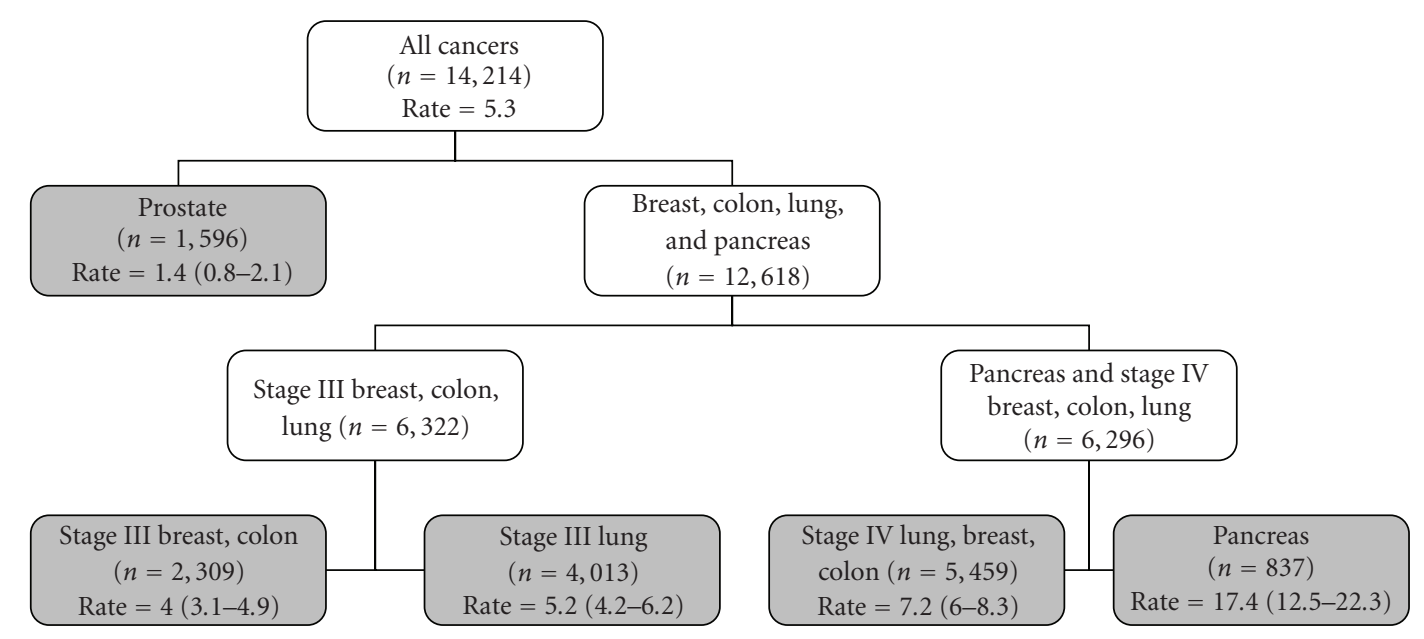

FIgURE 3: Recursive partitioning final analysis. Cancer of the prostate and pancreas is “nonlocalized." Rates are number of VTEs per 100 person-years (95\% confidence interval).

TABLE 3: Factors associated with developing a VTE within one year of cancer diagnosis: final cox proportional hazards model.

\begin{tabular}{lcccc}
\hline Variable & Hazard & \multicolumn{2}{c}{$95 \%$ CI } & $P$ value \\
\hline Prostate & 1.0 & & & \\
Breast & 4.86 & 2.93 & 8.08 & $<.01$ \\
Female colon & 6.60 & 3.83 & 11.38 & $<.01$ \\
Male colon & 3.73 & 2.10 & 6.62 & $<.01$ \\
Female lung & 7.57 & 4.67 & 12.26 & $<.01$ \\
Male lung & 7.64 & 4.73 & 12.34 & $<.01$ \\
Female pancreas & 21.57 & 12.21 & 38.09 & $<.01$ \\
Male pancreas & 17.68 & 9.48 & 32.95 & $<.01$ \\
Stage 3 & 1.0 & & & \\
Stage 4 & 1.75 & 1.44 & 2.12 & $<.01$ \\
Chemotherapy & 1.31 & 1.10 & 1.57 & $<.01$ \\
\hline
\end{tabular}

The higher risk for VTE in female compared with male colon cancer patients was unanticipated. Hormonal differences could play a significant role, but this is unlikely given the mean age in this study. There was also no difference in cancer-specific treatment between the sexes in this database. One study has shown that hospitalized men are $21 \%$ more likely than women to receive DVT prophylaxis, but it is unlikely that many men were given outpatient prophylaxis preferentially over women in this cohort [34]. A large database study by Khorana et al. demonstrated overall increased risk of VTE associated with female gender with an adjusted odds ratio of 1.14 (95\% CI 1.12 to 1.16) [35]. Conversely, Kröger et al. found no difference based on gender in their study of VTE risk factors specific to cancer patients [36]. Our finding of gender differences in VTE risk must be interpreted with caution as three gender-cancer comparisons were made. A $P$ value of .02 for the interaction term is of marginal significance, and additional studies are needed to confirm these results.
Although increasing age, CHF, COPD, dementia, and atrial fibrillation were protective in bivariate analysis, all were nonsignificant in multivariate analysis. Discordant results on the risk associated with age were shown in the studies by Khorana et al. [35] (adjusted odds ratio of 1.08 (1.05-1.11) for age > 65) and Kröger et al. [36] (no significant difference with age). Atrial fibrillation could appear protective because of concomitant use of anticoagulation. Older age, CHF, and COPD have been shown to increase the risk for VTE in the general population [37-39], and Chew and colleagues have demonstrated variable increased risk associated with increasing comorbidity in several different cancer populations [40-42]. Comorbidity codes may not be complete for every patient in the SEER-Medicare Database, which could have influenced our findings. Clinically, however, there may also be a real a hesitation to hospitalize older patients with both advanced cancer and other severe comorbidities who then develop VTE. As such, patients with more aggressive tumors may be less likely to undergo diagnostic evaluation for potential VTE if they face an extremely short life expectancy from their advanced cancer. Families may be more apt to consider end-of-life plans during these situations.

There are some limitations to consider. These are retrospective data using Medicare claims (ICD-9 coded) for the diagnosis of VTE and other comorbidities. Others have validated the accuracy of this approach, but many cases of VTE were likely missed or miscoded [6, 10, 43]. As a result, these VTE rates likely underestimate the true burden of disease in the cancer patient population. Our database also lacked specific laboratory information, such as platelet count, and does not specify which chemotherapeutic agents and doses were used. Importantly, the cohort was predominantly Caucasian. Given this fact, and the need to restrict the cohort to those at least 67 years old in order to appropriately use the SEER-Medicare Database, our results apply to older patients with cancer and are not necessarily generalizable to a more racially diverse and younger population. 
Only VTE claims associated with hospital admissions were considered to focus on the most clinically relevant and acute cases. Earlier studies demonstrated the effectiveness of outpatient VTE treatment using low-molecular-weight heparin (LMWH), but the first LMWH approved for this indication, enoxaparin, was toward the end of our study period in December, 1998 [44-46]. We found no studies that specifically evaluated national trends in outpatient treatment of VTE prior to 2000. While several studies assessed the utility of outpatient management of VTE toward the end of our study period [45, 47-49], there was disagreement in the literature about appropriate eligibility criteria for initial outpatient treatment, and outpatient treatment of PE was considered by some to be contraindicated [50, 51]. Given this and the absence of data describing large numbers of advanced cancer patients with incident VTE being treated as outpatients, the idea that the vast majority of these patients would have been admitted for standard-of-care treatment is valid.

Lastly, we have not validated the recursive partitioning approach used to stratify this cohort into clinically relevant risk groups. Our purpose for using RPA was to demonstrate that effective VTE risk stratification is potentially feasible even in this high-risk group with metastatic disease. As demonstrated by Khorana et al. using a multivariate model, stratification tools should undergo validation with separate data sets prior to implementation [52].

The combined one-year cumulative incidence of VTE in this study was $3.4 \%$. This is similar to rates seen in controls for trials of both LMWH and low-intensity coumadin as primary prophylaxis in patients with cancer, $3.3 \%$ and $4.4 \%$, respectively $[13,53]$. In high-risk medical patients without cancer, the PREVENT, ARTEMIS, and MEDENOX placebo-controlled trials revealed decrements in VTE rates with thromboprophylaxis from $5.0 \%$ to $2.8 \%, 10.5 \%$ to $5.6 \%$, and $14.9 \%$ to $5.5 \%$, respectively [54-56]. Higher baseline rates in the latter two trials are explained by their use of routine screening with Doppler ultrasound and/or venography. Without this sensitive screening method, we have demonstrated VTE rates in advanced cancer other than prostate ranging from 4.0 to 17.4 VTEs per 100 personyears. Future research should focus on the demonstration of VTE risk reduction in ambulatory, nonhospitalized cancer patients at highest risk.

One study that attempted to address VTE reduction in high risk cancer patients was the FAMOUS trial, which evaluated 374 patients with stages III and IV cancers of the breast, lung, gastrointestinal tract, pancreas, liver, genitourinary tract, ovary, and uterus [13]. No statistically significant survival benefit was noted with LMWH versus placebo after one year. A nonsignificant decrease in VTE rate from $3.3 \%$ to $2.4 \%$ was demonstrated, though diagnostic method was not specified and VTE rate was not a primary outcome. As a result, guidelines do not recommend primary VTE prophylaxis for nonhospitalized cancer patients in the absence of other risk factors [28]. A one-year event rate of $3.3 \%$ in the placebo group of the FAMOUS trial appears low compared to rates of 4.0 to17.4 per 100 person-years as seen in the highest-risk groups of the current study. A significant difference in event rates may be detected in future trials if the study groups are more homogeneously high-risk for VTE. As a case in point, a placebo-controlled trial using low-intensity coumadin in 311 women with stage IV breast cancer undergoing chemotherapy for a mean duration of six months did demonstrate a significant decrease in VTE rate from $4.4 \%$ to $0.7 \%$, which was the primary outcome for the trial [53]. The conclusion that VTE prophylaxis is relatively safe and effective in this group of patients was justified by the reduction in thrombosis rate, despite the fact that the secondary outcome of overall mortality reduction was not demonstrated within the follow-up period.

Our findings give additional insight about VTE risk in patients with cancer. We have shown that even in patients with advanced solid tumors, there is substantial variability in VTE risk. These data demonstrate that ambulatory cancer patients can be stratified into groups with VTE rates similar to nonsurgical, acutely ill-hospitalized patients. Additional trials are needed in oncology patient groups at the highest risk to determine if thromboprophylaxis is as effective as it is in acute medical illness. Most importantly, patients with advanced (stage IV) cancer of the lung, breast, colon, or "nonlocalized" pancreatic cancer as well as those patients undergoing chemotherapy should have close clinical monitoring for the development of VTE.

\section{Acknowledgments}

This study used the linked SEER-Medicare database. The interpretation and reporting of these data are the sole responsibility of the authors. The authors acknowledge the efforts of the Applied Research Program, NCI; the Office of Research, Development and Information, CMS; Information Management Services (IMS), Inc.; and the Surveillance, Epidemiology, and End Results Program tumor registries in the creation of the SEER-Medicare database. Dr. Gross's efforts were supported by a Beeson Career Development Award (1 K08 AG24842). Boehringer-Ingelheim provided funding to support the conduct of this study but was not involved with analysis of the data, drafting of the manuscript, or the decision to publish.

\section{References}

[1] P. M. Ridker, S. Z. Goldhaber, E. Danielson, et al., "Long-term, low-intensity warfarin therapy for the prevention of recurrent venous thromboembolism," The New England Journal of Medicine, vol. 348, no. 15, pp. 1425-1434, 2003.

[2] P. T. Vaitkus, A. Leizorovicz, A. T. Cohen, A. G. G. Turpie, C.-G. Olsson, and S. Z. Goldhaber, "Mortality rates and risk factors for asymptomatic deep vein thrombosis in medical patients," Thrombosis and Haemostasis, vol. 93, no. 1, pp. 7679, 2005.

[3] P. Prandoni, A. W. A. Lensing, A. Cogo, et al., "The long-term clinical course of acute deep venous thrombosis," Annals of Internal Medicine, vol. 125, no. 1, pp. 1-7, 1996.

[4] A. Ribeiro, P. Lindmarker, H. Johnsson, A. Juhlin-Dannfelt, and L. Jorfeldt, "Pulmonary embolism: one-year followup with echocardiography Doppler and five-year survival analysis," Circulation, vol. 99, no. 10, pp. 1325-1330, 1999. 
[5] C. C. Smith, L. I. Bernstein, R. B. Davis, D. M. Rind, and R. H. Shmerling, "Screening for statin-related toxicity: the yield of transaminase and creatine kinase measurements in a primary care setting," Archives of Internal Medicine, vol. 163, no. 6, pp. 688-692, 2003.

[6] H. K. Chew, T. Wun, D. Harvey, H. Zhou, and R. H. White, "Incidence of venous thromboembolism and its effect on survival among patients with common cancers," Archives of Internal Medicine, vol. 166, no. 4, pp. 458-464, 2006.

[7] C. P. Gross, D. H. Galusha, and H. M. Krumholz, "The impact of venous thromboembolism on risk of death or hemorrhage in older cancer patients," Journal of General Internal Medicine, vol. 22, no. 3, pp. 321-326, 2007.

[8] H. T. Sørensen, L. Mellemkjær, J. H. Olsen, and J. A. Baron, "Prognosis of cancers associated with venous thromboembolism," The New England Journal of Medicine, vol. 343, no. 25, pp. 1846-1850, 2000.

[9] A. A. Khorana, "Malignancy, thrombosis and Trousseau: the case for an eponym," Journal of Thrombosis and Haemostasis, vol. 1, no. 12, pp. 2463-2465, 2003.

[10] N. Levitan, A. Dowlati, S. C. Remick, et al., "Rates of initial and recurrent thromboembolic disease among patients with malignancy versus those without malignancy: risk analysis using medicare claims data," Medicine, vol. 78, no. 5, pp. 285291, 1999.

[11] J. W. Blom, C. J. M. Doggen, S. Osanto, and F. R. Rosendaal, "Malignancies, prothrombotic mutations, and the risk of venous thrombosis," Journal of the American Medical Association, vol. 293, no. 6, pp. 715-722, 2005.

[12] P. A. Thodiyil and A. K. Kakkar, "Variation in relative risk of venous thromboembolism in different cancers," Thrombosis and Haemostasis, vol. 87, no. 6, pp. 1076-1077, 2002.

[13] A. K. Kakkar, M. N. Levine, Z. Kadziola, et al., "Low molecular weight heparin, therapy with dalteparin, and survival in advanced cancer: the fragmin advanced malignancy outcome study (FAMOUS)," Journal of Clinical Oncology, vol. 22, no. 10, pp. 1944-1948, 2004.

[14] M. Karthaus, A. Kretzschmar, H. Kröning, et al., "Dalteparin for prevention of catheter-related complications in cancer patients with central venous catheters: final results of a doubleblind, placebo-controlled phase III trial," Annals of Oncology, vol. 17, no. 2, pp. 289-296, 2006.

[15] "Surveillance, Epidemiology, and End Results (SEER) Program SEER* Stat Database: Incidence-SEER 11 Regs PublicUse," November 2001 Sub (1992-1999). 2001, http://www.seer .cancer.gov/.

[16] B. Miller, L. Ries, and B. Hankey, Eds., Cancer Statistics Review 1973-1989, NIH, Bethesda, Md, USA, 1992.

[17] A. L. Potosky, G. F. Riley, J. D. Lubitz, R. M. Mentnech, and L. G. Kessler, "Potential for cancer related health services research using a linked Medicare-tumor registry database," Medical Care, vol. 31, no. 8, pp. 732-748, 1993.

[18] L. Ries, M. Eisner, C. Kosary, et al., SEER Cancer Statistics Review, 1975-2002, National Cancer Institute, Bethesda, Md, USA, 2005.

[19] D. K. Espey, X.-C. Wu, J. Swan, et al., "Annual report to the nation on the status of cancer, 1975-2004, featuring cancer in American Indians and Alaska Natives," Cancer, vol. 110, no. 10, pp. 2119-2152, 2007.

[20] C. N. Klabunde, A. L. Potosky, J. M. Legler, and J. L. Warren, "Development of a comorbidity index using physician claims data," Journal of Clinical Epidemiology, vol. 53, no. 12, pp. 1258-1267, 2000.
[21] M. Abdollahi, M. Cushman, and F. R. Rosendaal, "Obesity: risk of venous thrombosis and the interaction with coagulation factor levels and oral contraceptive use," Thrombosis and Haemostasis, vol. 89, no. 3, pp. 493-498, 2003.

[22] L. Friberg, N. Hammar, M. Ringh, H. Pettersson, and M. Rosenquist, "Stroke prophylaxis in atrial fibrillation: who gets it and who does not? Report from the Stockholm Cohortstudy on Atrial Fibrillation (SCAF-study)," European Heart Journal, vol. 27, no. 16, pp. 1954-1964, 2006.

[23] D. B. Badesch, V. F. Tapson, M. D. McGoon, et al., "Continuous intravenous epoprostenol for pulmonary hypertension due to the scleroderma spectrum of disease: a randomized, controlled trial," Annals of Internal Medicine, vol. 132, no. 6, pp. 425-434, 2000.

[24] E. B. Lamont, D. S. Lauderdale, R. L. Schilsky, and N. A. Christakis, "Construct validity of medicare chemotherapy claims: the case of 5FU," Medical Care, vol. 40, no. 3, pp. 201211, 2002.

[25] D. Schrag, S. E. Gelfand, P. B. Bach, J. Guillem, B. D. Minsky, and C. B. Begg, "Who gets adjuvant treatment for stage II and III rectal cancer? Insight from surveillance, epidemiology, and end results-medicare," Journal of Clinical Oncology, vol. 19, no. 17, pp. 3712-3718, 2001.

[26] G. M. Freedman, A. L. Hanlon, B. L. Fowble, P. R. Anderson, and N. Nicoloau, "Recursive partitioning identifies patients at high and low risk for ipsilateral tumor recurrence after breast-conserving surgery and radiation," Journal of Clinical Oncology, vol. 20, no. 19, pp. 4015-4021, 2002.

[27] H. P. Zhang and B. Singer, Eds., Recursive Partitioning in the Health Sciences, Springer, New York, USA, 1999.

[28] W. H. Geerts, G. F. Pineo, J. A. Heit, et al., "Prevention of venous thromboembolism: the Seventh ACCP Conference on Antithrombotic and Thrombolytic Therapy," Chest, vol. 126, supplement 3, pp. 338S-400S, 2004.

[29] J. H. Beer, A. Haeberli, A. Vogt, et al., "Coagulation markers predict survival in cancer patients," Thrombosis and Haemostasis, vol. 88, no. 5, pp. 745-749, 2002.

[30] S. R. Deitcher, "Cancer-related deep venous thrombosis: clinical importance, treatment challenges, and management strategies," Seminars in Thrombosis and Hemostasis, vol. 29, no. 3, pp. 247-258, 2003.

[31] S. R. Deitcher, J. K. Erban, and S. A. Limentani, "Acquired free protein $\mathrm{S}$ deficiency associated with multiple myeloma: a case report," American Journal of Hematology, vol. 51, no. 4, pp. 319-323, 1996.

[32] S. Sallah, J. Y. Wan, and N. P. Nguyen, "Venous thrombosis in patients with solid tumors: determination of frequency and characteristics," Thrombosis and Haemostasis, vol. 87, no. 4, pp. 575-579, 2002.

[33] M. Cushman, A. W. Tsai, R. H. White, et al., "Deep vein thrombosis and pulmonary embolism in two cohorts: the longitudinal investigation of thromboembolism etiology," American Journal of Medicine, vol. 117, no. 1, pp. 19-25, 2004.

[34] N. Kucher, V. F. Tapson, R. Quiroz, et al., "Gender differences in the administration of prophylaxis to prevent deep venous thrombosis," Thrombosis and Haemostasis, vol. 93, no. 2, pp. 284-288, 2005.

[35] A. A. Khorana, C. W. Francis, E. Culakova, N. M. Kuderer, and G. H. Lyman, "Frequency, risk factors, and trends for venous thromboembolism among hospitalized cancer patients," Cancer, vol. 110, no. 10, pp. 2339-2346, 2007.

[36] K. Kröger, D. Weiland, C. Ose, et al., "Risk factors for venous thromboembolic events in cancer patients," Annals of Oncology, vol. 17, no. 2, pp. 297-303, 2006. 
[37] R. Alikhan, F. Peters, R. Wilmott, and A. T. Cohen, "Fatal pulmonary embolism in hospitalised patients: a necropsy review," Journal of Clinical Pathology, vol. 57, no. 12, pp. 1254 $1257,2004$.

[38] J. A. Heit, W. M. O'Fallon, T. M. Petterson, et al., "Relative impact of risk factors for deep vein thrombosis and pulmonary embolism: a population-based study," Archives of Internal Medicine, vol. 162, no. 11, pp. 1245-1248, 2002.

[39] M. Ambrosetti, W. Ageno, A. Spanevello, M. Salerno, and R. F. E. Pedretti, "Prevalence and prevention of venous thromboembolism in patients with acute exacerbations of COPD," Thrombosis Research, vol. 112, no. 4, pp. 203-207, 2004.

[40] A. Alcalay, T. Wun, V. Khatri, et al., "Venous thromboembolism in patients with colorectal cancer: incidence and effect on survival," Journal of Clinical Oncology, vol. 24, no. 7, pp. 1112-1118, 2006.

[41] H. K. Chew, A. M. Davies, T. Wun, D. Harvey, H. Zhou, and R. H. White, "The incidence of venous thromboembolism among patients with primary lung cancer," Journal of Thrombosis and Haemostasis, vol. 6, no. 4, pp. 601-608, 2008.

[42] H. K. Chew, T. Wun, D. J. Harvey, H. Zhou, and R. H. White, "Incidence of venous thromboembolism and the impact on survival in breast cancer patients," Journal of Clinical Oncology, vol. 25, no. 1, pp. 70-76, 2007.

[43] D. C. Hsia, W. M. Krushat, A. B. Fagan, J. A. Tebbutt, and R. P. Kusserow, "Accuracy of diagnostic coding for Medicare patients under the prospective-payment system," The New England Journal of Medicine, vol. 318, no. 6, pp. 352-355, 1988.

[44] M. A. Koopmanschap and F. F. Rutten, "The impact of indirect costs on outcomes of health care programs," Health Economics, vol. 3, no. 6, pp. 385-393, 1994.

[45] M. Levine, M. Gent, J. Hirsh, et al., "A comparison of lowmolecular-weight heparin administered primarily at home with unfractionated heparin administered in the hospital for proximal deep-vein thrombosis," The New England Journal of Medicine, vol. 334, no. 11, pp. 677-681, 1996.

[46] FDA, "FDA Website," July 2005, http://www.accessdata.fda .gov/scripts/cder/drugsatfda/index.cfm.

[47] M. M. W. Koopman, P. Prandoni, F. Piovella, et al., "Treatment of venous thrombosis with intravenous unfractionated heparin administered in the hospital as compared with subcutaneous low-molecular-weight heparin administered at home," The New England Journal of Medicine, vol. 334, no. 11, pp. 682-687, 1996.

[48] G. Simonneau, H. Sors, B. Charbonnier, et al., "A comparison of low-molecular-weight heparin with unfractionated heparin for acute pulmonary embolism. The THESEE Study Group. Tinzaparine ou Heparine Standard: Evaluations dans l'Embolie Pulmonaire," The New England Journal of Medicine, vol. 337, no. 10, pp. 663-669, 1997.

[49] P. S. Wells, M. J. Kovacs, J. Bormanis, et al., "Expanding eligibility for outpatient treatment of deep venous thrombosis and pulmonary embolism with low-molecular-weight heparin: a comparison of patient self-injection with homecare injection," Archives of Internal Medicine, vol. 158, no. 16, pp. 1809-1812, 1998.

[50] P. S. Wells, "Outpatient treatment of patients with deepvein thrombosis or pulmonary embolism," Current Opinion in Pulmonary Medicine, vol. 7, no. 5, pp. 360-364, 2001.

[51] R. D. Yusen, B. M. Haraden, B. F. Gage, R. S. Woodward, B. G. Rubin, and M. D. Botney, "Criteria for outpatient management of proximal lower extremity deep venous thrombosis," Chest, vol. 115, no. 4, pp. 972-979, 1999.
[52] A. Khorana, N. M. Kuderer, E. Culakova, G. H. Lyman, and C. W. Francis, "Development and validation of a predictive model for chemotherapy- associated thrombosis," Blood, vol. 111, no. 10, pp. 4902-4907, 2008.

[53] M. Levine, J. Hirsh, M. Gent, et al., "Double-blind randomised trial of very-low-dose warfarin for prevention of thromboembolism in stage IV breast cancer," The Lancet, vol. 343, no. 8902, pp. 886-889, 1994.

[54] A. Hurria, M. S. Lachs, H. J. Cohen, H. B. Muss, and A. B. Kornblith, "Geriatric assessment for oncologists: rationale and future directions," Critical Reviews in Oncology/Hematology, vol. 59, no. 3, pp. 211-217, 2006.

[55] A. Leizorovicz, A. T. Cohen, A. G. G. Turpie, C.-G. Olsson, P. T. Vaitkus, and S. Z. Goldhaber, "Randomized, placebocontrolled trial of dalteparin for the prevention of venous thromboembolism in acutely ill medical patients," Circulation, vol. 110, no. 7, pp. 874-879, 2004.

[56] M. M. Samama, A. T. Cohen, J.-Y. Darmon, et al., "A comparison of enoxaparin with placebo for the prevention of venous thromboembolism in acutely ill medical patients. Prophylaxis in Medical Patients with Enoxaparin Study Group," The New England Journal of Medicine, vol. 341, no. 11, pp. 793-800, 1999. 


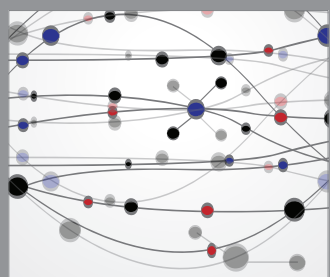

The Scientific World Journal
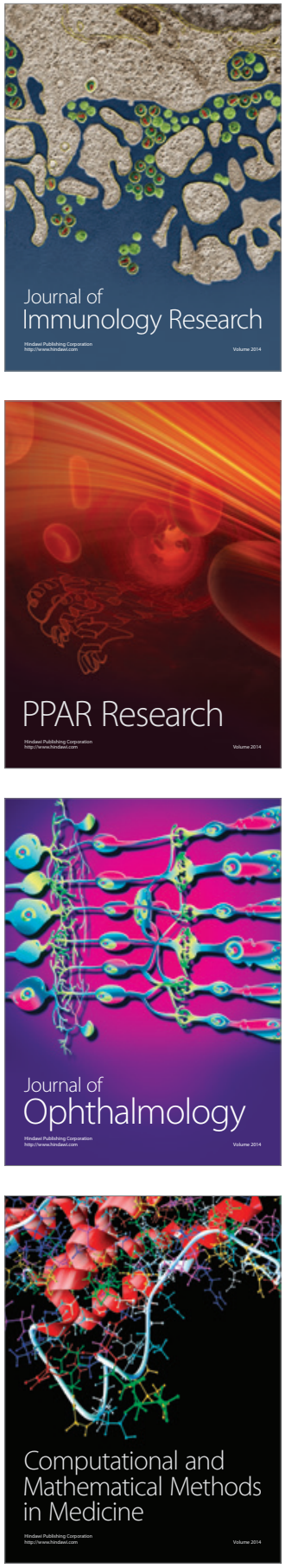

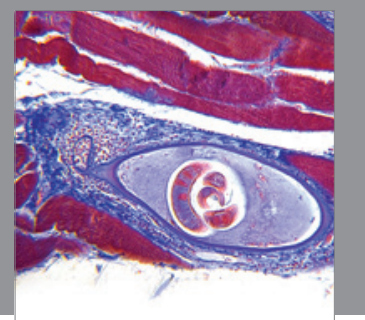

Gastroenterology

Research and Practice
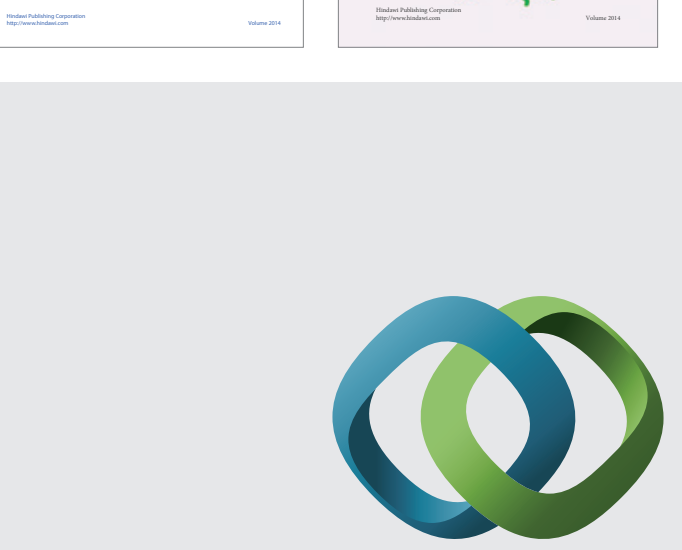

\section{Hindawi}

Submit your manuscripts at

http://www.hindawi.com
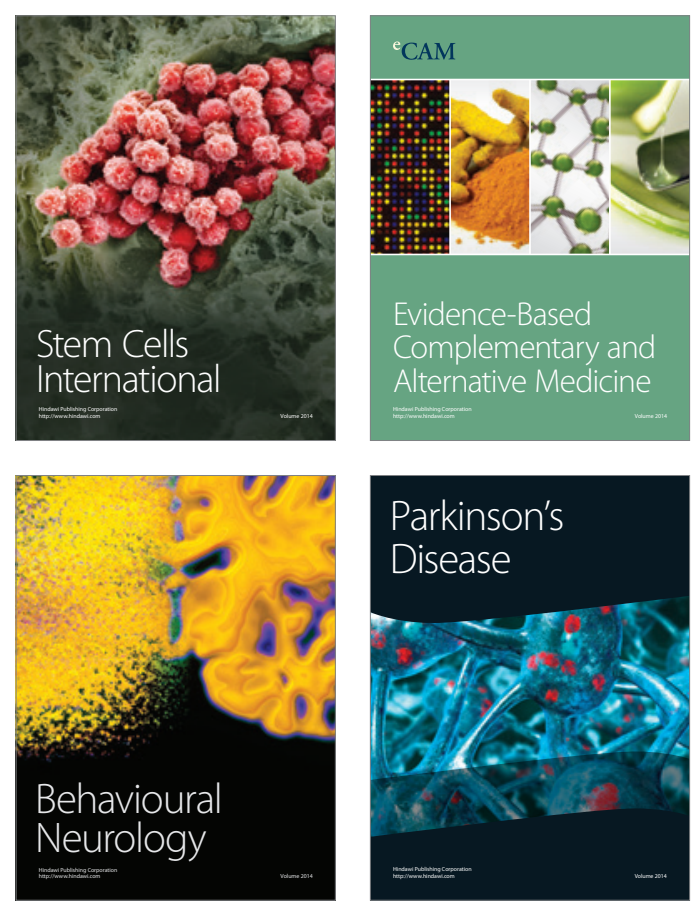

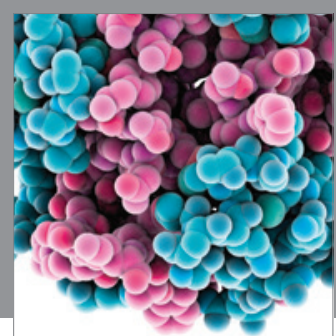

Journal of
Diabetes Research

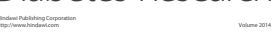

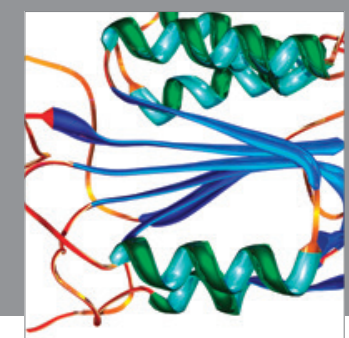

Disease Markers
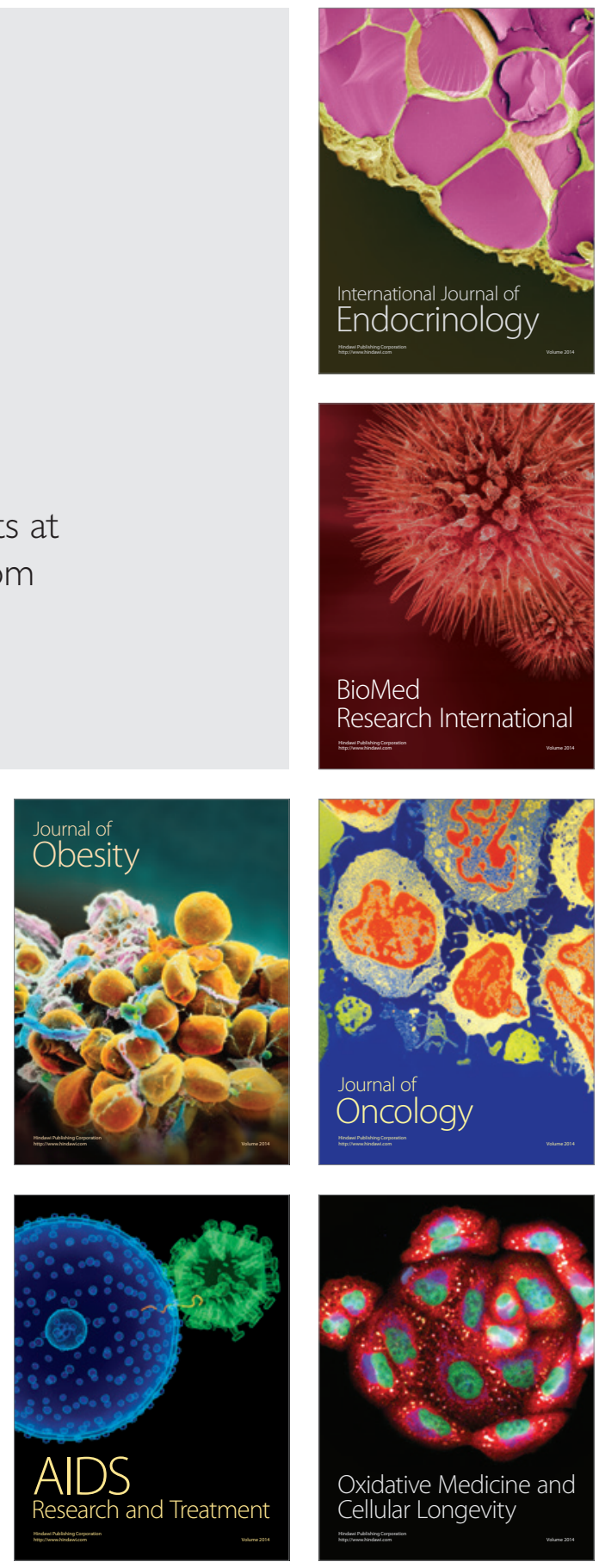